\title{
RESIDUAL EQUISINGULARITY
}

\author{
JOSEPH BECKER AND JOHN STUTZ
}

\begin{abstract}
Let $V$ be a complex analytic set and $\operatorname{Sg} V$ the singular set of $V$ be in codimension one; then the set of points of $\operatorname{Sg} V$ for which $V$ is not residually equisingular along $\operatorname{Sg} V$ is a proper analytic subset of $\operatorname{Sg} V . V$ is said to be residually equisingular along $\mathrm{Sg} V$ if all one dimensional slices of $V$ transverse to $\mathrm{Sg} V$ have isomorphic resolutions.
\end{abstract}

Let $V$ be an analytic subset of a domain $U \subset \mathbf{C}^{n}$ of pure dimension $r$ and let $\operatorname{Sg} V$, the singular locus of $V$, be of dimension $r-1$. For $p \in \operatorname{Sg} V$ such that near $p, \operatorname{Sg} V$ is an $(r-1)$-dimensional manifold, the second author introduced [3] three different notions of equisingularity of $V$ along $\operatorname{Sg} V$ at $p$ : weak, strong, and residual. These generalize various portions of the theory of equisingularity for hypersurfaces developed by Zariski [5]. The results pertaining to residual equisingularity were published jointly in [1]. The purpose of this note is to improve one of the results in [1]. There we showed that if $V$ is weakly equisingular along $\operatorname{Sg} V$ at $p$, then near $p\{q \in \operatorname{Sg} V: V$ is not residually equisingular along $\operatorname{Sg} V$ at $q$ \} is contained in an analytic subset of $\operatorname{Sg} V$ of dimension $<r-1$. We improve this showing that $\{q \in \operatorname{Sg} V: V$ is not residually equisingular along $\operatorname{Sg} V$ at $q\}$ is an analytic subset of $\operatorname{Sg} V$ of dimension $<r-1$. In the course of our proof we will clarify our original definition of residual equisingularity and develop an alternative formulation which does not depend on the imbedding of $V$ in $U$.

The authors would like to thank Professor Joseph Lipman for suggesting the technique employed here.

Let $p, \operatorname{Sg} V$ and $V$ be as above. Replacing $U$ by a suitable neighborhood of $p$ we may select coordinates $\left(x_{i}\right)$ such that $p$ becomes the origin, $\operatorname{Sg} V$ $=\left\{q \in U: q_{i}=0\right.$ for $\left.i \geqslant r\right\}$, and for $q \in \operatorname{Sg} V, V_{q}=\left\{x \in V: x_{i}=q_{i}\right.$ for $i<r\}$ is one dimensional. Such coordinates will be said to be adapted to $V$ at $p$. Each $V_{q}$ can be resolved by a sequence of local quadratic transformations, however the series may vary greatly with $q ; V$ is said to be residually equisingular (see Definitions 1 and 2) if the series does not vary. For such coordinates, and for $W, W^{\prime}$ appropriate open subsets of $V$, we define 3 types of maps from $W$ to $W^{\prime}$ :

(i) $T(x)=\left(x_{1}, \ldots, x_{r}, x_{r+1}-t_{1}(x), \ldots, x_{n}-t_{n-r}(x)\right)$ where the $t_{i}$ are holomorphic functions of $x_{1}, \ldots, x_{r}$ alone.

(ii) $S(x)=\left(x_{1}, \ldots, x_{r-1}, s_{0}(x), \ldots, s_{n-r}(x)\right)$ where $s_{i}(x)=\sum_{j=r}^{n} a_{i j} x_{j}, a_{i, j}$ $\in \mathbf{C}$, and $r k\left(a_{i, j}\right)=n-r$.

Received by the editors March 12, 1975.

AMS (MOS) subject classifications (1970). Primary 32C45; Secondary 14B05.

Key words and phrases. Residual equisingularity, local quadratic transformation, analytic variety, resolution. 
(iii) $Q(x)=\left(x_{1}, \ldots, x_{r}, x_{r} x_{r+1}, \ldots, x_{r} x_{n}\right)$.

Definition 1. A connected sequence of local quadratic transformations is a sequence of maps $f_{j}: W_{j} \rightarrow W_{j-1}, i=1, \ldots, l$, such that:

(a) $F_{j}=f_{1} \circ f_{2} \circ \cdots \circ f_{j}$ is a proper modification of $W_{0}$, that is $F_{j}^{-1}$ (compact set) is compact and $F_{j}$ is biholomorphic on the inverse image of $\operatorname{Reg} W_{0}$, the regular points of $W_{0}$, and $F_{j}^{-1}\left(\operatorname{Reg} W_{0}\right)$ is dense in $W_{j}$.

(b) $f_{j}$ is the restriction of a map of type (i), (ii) or (iii) on each component $W_{j, k}$ of $W_{j}$.

(c) If $f_{j}\left(W_{j, k_{1}}\right) \cap f_{j}\left(W_{j, k_{2}}\right)$ is nonnull then $f_{j} \mid W_{j, k_{1}}$ and $F \mid W_{j, k_{2}}$ are of the same type.

Suppose that a connected sequence of quadratic transformations is given and the image of $F_{j}$ is a neighborhood of $p$. Set $V_{j}=F_{j}^{-1}(V)$ (of course if a type (iii) map occurs, we omit those components which are contained in $\left.\left\{x \in U: x_{r}=0\right\}\right)$.

Definition 2. $V$ is residually equisingular along $\operatorname{Sg} V$ at $p$ if there is a connected sequence of quadratic transformations as above such that:

(a) $F_{l}: V_{l} \rightarrow V$ is a resolution;

(b) $F_{l}^{-1}(\mathrm{Sg} V)$ is an $r-1$ manifold and $F_{l}$ restricted to each component of it is biholomorphic.

The above definition appears weaker than that given in [1]. There we required that given any $q \in F_{j_{0}}^{-1}(p)$ and $V_{j_{0}, k}$ a branch of $V_{j_{0}}$ at $q$ that either $V_{j_{0}, k}$ is nonsingular at $q$ or $\mathrm{Sg} V_{j_{0}, k}=F_{j_{0}}^{-1}(\mathrm{Sg} V)$ near $q$. This condition is actually a consequence of our other conditions. To see this assume there is a $q$ at which $\operatorname{Sg} V_{j_{0}, k}$ is a proper subset of $F_{j_{0}}^{-1}(\operatorname{Sg} V)$; then $\operatorname{dim}_{q} \operatorname{Sg} V_{j_{0}, k}<r-1$. Since the maps $\left(f_{j}\right)^{-1}$ are weakly holomorphic the singular loci of the portions of the $V_{j}, j>j_{0}$, lying over $V_{j_{0}+1, k}$ are also of dimension $<r-1$. Thus it suffices to consider the case where $V_{j_{0}+1, k}$ is nonsingular and $f_{j_{0}+1} \mid V_{j_{0}+1, k}$ is of type (iii). Now as in the first portion of the proof of Proposition 1 in [1], one sees that $\operatorname{dim} C_{4}\left(V_{j_{0}+1, k}, q\right)=r$; and [2, Proposition 1.8] $\operatorname{dim} C_{4} V=\operatorname{dim} V$, codim $\operatorname{Sg} V \geqslant 2$ implies $V$ nonsingular.

DEFINITION 3. Let $X$ be an analytic variety and $g$ a coherent sheaf of ideals in $X_{X} \mathcal{O}$, the reduced sheaf of homolomorphic functions on $X,{ }_{n} \mathcal{O} / I(V)$ where $I$ is self-radical. Then the blow-up of $X$ along $g, B_{J}(X)$, is defined as follows: let $g_{1}, \ldots, g_{m}$ generate $g$ over $X \mathcal{O}, Y=\operatorname{support} \mathcal{G}$, and $B_{J}(X)$ the closure in $X \times \mathbf{C P}^{m-1}$ of $\left\{(x, z) \in(X-Y) \times \mathbf{C P}^{m-1}: z_{i} g_{j}(x)=z_{j} g_{i}(x)\right.$ all $\left.i, j\right\}$. Then the natural projection $\pi: B_{J}(X) \rightarrow X$ is a proper modification of $X$. This construction is independent (up to analytic isomorphism) of the choice of generators of $g$ and of the coordinates on $\mathbf{C}^{n}$.

Now let $g$ be the ideal sheaf of $\operatorname{Sg} X$ and $B_{s}(X)$ denote the blow-up of $X$ along $g$. We will see there is a one-to-one correspondence between local quadratic transforms of $X$ and blow-ups such that $\pi^{-1}(\operatorname{Sg}(X)) \rightarrow \operatorname{Sg} X$ is a biholomorphism on each component.

Let $p \in \operatorname{Reg}(\operatorname{Sg}(X)),\left(x_{i}\right)$ coordinates adapted to $X$ at $p$, and $Q: X^{\prime} \rightarrow X$ a local quadratic transform of the third type $Q\left(x_{1}, \ldots, x_{n}\right)=\left(x_{1}, \ldots, x_{r}\right.$, $\left.x_{r} x_{r+1}, \ldots, x_{r} x_{n}\right)=\left(y_{1}, \ldots, y_{n}\right)$. Now $I(\operatorname{Sg} V)$ is generated by $x_{r}, x_{r+1}$, $\ldots, x_{n}$, so form the blow-up $B_{s}(X) \subset\left\{(y, z) \in X \times \mathbf{C P}^{n-r}: y_{i} z_{j}=y_{j} z_{i}\right.$ for $i, j \geqslant r\}$. There is an injection $i: X^{\prime} \rightarrow B_{s}(X)$,

$$
i\left(x_{1}, \ldots, x_{n}\right)=\left(x_{1}, \ldots, x_{r}, x_{r} x_{r+1}, \ldots, x_{r} x_{n} ; 1, x_{r+1}, \ldots, x_{n}\right)
$$


such that $Q=\pi i$. Since $B_{s}(X) \subset\left\{(y, z): z_{r} \neq 0\right\}, \beta: B_{s}(X) \rightarrow X^{\prime}, \beta(x, y)$ $=x$ is the inverse to $i$. Since $Q$ is unramified over $\operatorname{Sg} X, \pi$ is also.

Conversely if $\pi: B_{s}(X) \rightarrow X$ is unramified over $\operatorname{Sg} X$, one can define an inverse $\varphi: \operatorname{Sg} X \rightarrow B_{s}(X)$ of $\pi$ and by shrinking the neighborhood of $p$, assume that some $Z_{i}$ (say $Z_{r}$ ) is nonzero on the image of $\varphi$. Let

$$
Q\left(y_{1}, \ldots, y_{n}\right)=\left(y_{1}, \ldots, y_{r}, y_{r} y_{r+1}, \ldots, y_{r} y_{n}\right)
$$

and $X^{\prime}=Q^{-1}(X)$; there is an analytic equivalence $\rho: B_{s}(X) \rightarrow X^{\prime}$ defined by

$$
\rho\left(x_{1}, \ldots, x_{n}, z_{r}, \ldots, z_{n}\right)=\left(x_{1}, \ldots, x_{r}, z_{r+1} / z_{r}, \ldots, z_{n} / z_{r}\right)
$$

such that $\pi=Q \rho . Q$ is unramified over $\operatorname{Sg} V$, since $\pi$ is too.

For each integer $j>0$ we can define a space $B_{j}$ and a map $g_{j}: B_{j} \rightarrow V$ as follows: $\pi_{1}: B_{1} \rightarrow V$ is the blow-up of $V$ along $\operatorname{Sg} V$. For $j>1$, set $S_{j}$ $=g_{j}^{-1}(\operatorname{Sg} V)$, let $\pi_{j}: B_{j+1} \rightarrow B_{j}$ be the blow-up of $B_{j}$ along $S_{j}$, and set $g_{j+1}=\pi_{j} \circ g_{j}$.

LEMMA. The following are equivalent:

(a) $V$ is residually equisingular along $\operatorname{Sg} V$ at $p \in \operatorname{Sg} V$.

(b) If one replaces $U$ by a suitable neighborhood of $p$, then there is an integer $m>0$ such that $B_{m}$ is nonsingular, and for $j \leqslant m, S_{j}$ is an $(r-1)$-dimensional manifold, and $\pi_{j}$ restricted to each component of $S_{j}$ is biholomorphic.

Proof. Follows immediately from the above and the fact that types (i) and (ii) maps are biholomorphic.

Proposition. The set $A$ of points $p$ such that $V$ is not residually equisingular along $\operatorname{Sg} V$ at $p$ is an analytic subset of $V$.

Proof. Take a succession of blow-ups of $V$ along the singular locus; define inductively $V_{0}=V, V_{i+1}=B_{\mathrm{Sg} V_{i}}\left(V_{i}\right), \pi_{i}: V_{i} \rightarrow V_{i-1}, g_{j}=\pi_{1} \circ \cdots \circ \pi_{j}$ and $S_{i}$
$=g_{i}^{-1}(\operatorname{Sg} V)$.

Since $A$ is a nowhere dense set in $\operatorname{Sg} V$ [1, Proposition 2], there exists some $l>0$ such that $\operatorname{codim}_{V_{l}} \operatorname{Sg} V_{l} \geqslant 2$. Then, we have that

$$
A=g_{l}\left(\operatorname{Sg} B_{l}\right) \cup\left(\bigcup_{j} g_{j}\left(\operatorname{Sg} S_{j} \cup\left\{q \in S_{j}-\operatorname{Sg} S_{j}: r k_{q} g_{j} \mid S_{j}<r-1\right\}\right)\right)
$$

is the finite union of images of analytic sets under proper maps.

In addition to providing a characterization of residual equisingularity, the Lemma also provides the justification for a simple method of testing for residual equisingularity in particular examples. As observed in [3], since residual equisingularity implies weak equisingularity, all potential equisingular points have Puiseux series normalizations, so one can attempt to check for residual equisingularity by direct calculation. For example let $V$ be the image of the map $f: \mathbf{C}^{2} \rightarrow \mathbf{C}^{4}$ given by $f(x, y)=\left(x, y^{9}, y^{7}, x y^{8}\right)$. (See [1, Example 2] for more details on this example.) The obvious attempt to construct the required connected sequence of local quadratic transformations leads to a dead end. However, initially one does not know that some other sequence will not work. By constructing from the initial sequence the blow-ups $B_{1}, B_{2}$, one sees that $\operatorname{dim} \pi_{2}^{-1}(0)=1$, so $V$ is not residually equisingular at 0 . 
In the classical theory of plane curves, one has the equivalence of characteristic pairs and multiplicities $e_{i}$ of the canonical sequence of blow-ups which resolve the singularity of an irreducible plane curve. These determine the length of the conductor $\operatorname{dim}_{\mathbf{C}} \tilde{\mathcal{\theta}} / \mathcal{O}$, and the, exponent of the conductor on the normalization $\operatorname{dim}_{\mathbf{C}} \tilde{\mathcal{\theta}} / \mathcal{O}$, via the Italian Geometers formula:

$$
\exp =2 \cdot \text { length }=\sum e_{i}\left(e_{i}-1\right) .
$$

For equisingularity in codim one [5], these results extend as follows: $V$ is equisingular along $\operatorname{Sg} V$ if and only if the curves have the same multiplicity sequence. Furthermore, if the length of the conductor is the same for each curve, then $V$ is equisingular along $\operatorname{Sg} V$.

For equisingularity in higher codim, this all breaks down. Neither the exponent or length is constant, and it is not true that one thing is twice the other.

EXAmple. Let $V$ be the image in $\mathbf{C}^{4}$ of $(s, t) \rightarrow\left(s, t^{3}, t^{4}, s t^{5}\right)$. Then $V$ is residually and strongly equisingular. For $s \neq 0$, exponent $=3$, length $=2$, and $\sum e_{i}\left(e_{i}-1\right)=6$. For $s=0$, exponent $=6$, length $=3$, and $\sum e_{i}\left(e_{i}-1\right)$ $=6$.

However, one can easily see from looking at the Puiseux expansions the multiplicity sequence is the same for each curve in a residually equisingular variety and so $\sum e_{i}\left(e_{i}-1\right)$ is constant. Recently Fischer [4] has found a possible interpretation for this number: let $C$ be the complete local ring of a irreducible curve, $\bar{C}$ its integral closure in its field of quotients, and $I_{C}$ the high order differentials of $\bar{C}$ over $C$. Then length $I_{C}=\sum e_{i}\left(e_{i}-1\right)$ and $I_{C_{1}} \simeq I_{C_{2}}$ as $\bar{C}$ modules if and only if $C_{1}$ and $C_{2}$ have the same multiplicity sequence.

REMARK. Strong equisingularity does not even imply that the multiplicity sequence is constant. This can be seen from the example $(s, t) \rightarrow\left(s, t^{5}, t^{7}, s t^{8}\right)$.

\section{REFERENCES}

1. J. Becker and J. Stutz, Resolving singularities via local quadratic transformations, Rice Univ. Studies 59 (1973), 1-9.

2. J. Stutz, Analytic sets as branched coverings, Trans. Amer. Math. Soc. 166 (1972), 241-259. MR 48 \#2420.

3. - Equisingularity and equisaturation in codimension 1, Amer. J. Math. 94 (1972), $1245-1268$.

4. K. Fischer, The module decomposition of $I(\bar{A} / A)$, Trans. Amer. Math. Soc. 186 (1973), 113-128.

5. O. Zariski, Studies in equisingularity. I, II, III, Amer. J. Math. 87 (1965), 507-536, 972-1006; ibid. 90 (1968), 961-1023. MR 31 \#2243; 33 \#125; 38 \#775.

Division of Mathematical Sciences, Purdue University, West Lafayette, Indiana 47907

Allen Center, State University of New York at Albany, Albany, New York 1222 